\begin{tabular}{ll}
\hline Frontiers \\
Mats at and \\
Manser
\end{tabular}

\title{
UNSTEADY MHD THREE-DIMENSIONAL CASSON NANOFLUID FLOW OVER A POROUS LINEAR STRETCHING SHEET WITH SLIP CONDITION
}

\author{
I.S. Oyelakin ${ }^{\mathrm{a},}$, S. Mondal ${ }^{\mathrm{a}, *}$, P. Sibanda ${ }^{\mathrm{a}}$ \\ ${ }^{a}$ School of Mathematics, Statistics and Computer Science, University of KwaZulu-Natal, Pietermaritzburg, Private Bag X01 Scottsville 3209, South \\ Africa \\ ${ }^{\dagger}$ DST-NRF Centre of Excellence in Mathematical and Statistical Sciences (CoE-MaSS), Private Bag 3, Wits 2050, Johannesburg, South Africa
}

\begin{abstract}
In this paper we study the effects of thermal radiation, heat and mass transfer on the unsteady magnetohydrodynamic(MHD) flow of a three dimensional Casson nanofluid. The flow is subject to partial slip and convective conditions. The traditional model which includes the effects of Brownian motion and thermophoresis is revised so that the nanofluid particle volume fraction on the boundary is not actively controlled. In this respect the problem is more realistic. The dimensionless governing equations were solved using the spectral quasi-linearisation method. This work aims to fill the gap in existing literature by showing the effects of porosity, magnetic field and stretching ratio parameter on the flow of the Casson Nanofluid model over a porous linearly stretching sheet with the incorporation of the nanoparticles on the concentration boundary condition. It is observed that increase in the unsteadiness of the flow tends to decrease the momentum, thermal and nanoparticles volume fraction profiles. The results are benchmarked with previously published results.
\end{abstract}

Keywords: 3D-Casson Nanofluid; MHD; Porous linear stretching sheet; Spectral quasi-linearisation method.

\section{INTRODUCTION}

The flow of fluid over a stretching surface occurs in a variety of engineering processes with practical applications in areas like copper spiralling, glass blowing, paper production, cord depiction, etc. Crane (1970) studied the convection boundary layer viscous flow over a linear stretching sheet and presented a closed form exponential solution of the model equations. The problem has since been extended to other aspects of nonNewtonian fluid, for instance, to include MHD effects and media porosity. Bhattacharyya (2013) focused on boundary layer stagnation-point flow of Casson fluid and heat transfer towards a shrinking/stretching sheet. Again, Kumar et al. (2015) examined thermal diffusion and radiation effects on unsteady free convection flow In the presence of magnetic field fixed relative to the fluid or the plate.

Khan and Pop (2010) investigated the problem of laminar fluid flow which results from the stretching of a flat surface in a nanofluid by analyzing the development of the steady boundary layer flow, heat transfer and nanoparticle fraction over a stretching surface in a nanofluid. Makinde and Aziz (2011) studied the boundary layer flow induced in a nanofluid due to a linearly stretching sheet by investigating the effect of a convective boundary condition on the boundary layer flow, heat transfer and nanoparticle fraction over a stretching surface. In the study, a convective boundary condition was employed instead of the usual thermal conditions of constant temperature or constant heat flux. Noghrehabadi et al. (2012a) examined the combined effects of Brownian motion, thermophoresis and magnetic field on the steady boundary-layer flow and heat transfer of nanofluids over a linear isothermal stretching sheet. In most studies, it is assumed that at a solid boundary, the fluid will have zero velocity relative to the boundary, but by considering Navier's condition, the velocity slip is assumed to be proportional to the local shear stress and this implies there is a linear relationship between the slip velocity and the shear stress at the wall. Slip effects analisis on the boundary layer flow and heat transfer over a stretching surface in the presence of nanoparticle fraction, by considering the boundary-layer heat transfer of nanofluids in the presence of Brownian motion and thermophoresis forces over a stretching sheet has been studied by Noghrehabadi et al. (2012b). Furthermore, Noghrehabadi et al. (2013) studied effect of the slip boundary condition on heat transfer characteristics of nanofluid flow over stretching sheet prescribed convective boundary conditions using a model that took into account the dynamic effects of nanoparticles. Their work showed that increasing the slip factor and the Biot number strongly influenced the reduced Nusselt and Sherwood numbers.

Casson fluid is a non-Newtonian fluid that behaves like an elastic solid at low shear strain and like a Newtonian fluid above a critical yield stress. Common examples of Casson fluids include tomato sauce and blood. Mustafa et al. (2011) reported on the flow and heat transfer of a

${ }^{*}$ Corresponding author's email id:sabya.mondal.2007@gmail.com 
Table 1 Comparison of values for skin friction coefficients for various values of $\beta$ and $\mathrm{M}$ with those of Nadeem et al. (2014)

\begin{tabular}{|c|c|c|c|c|c|c|c|c|c|}
\hline \multirow[t]{3}{*}{$\beta$} & \multirow{3}{*}{$\mathbf{M}$} & \multicolumn{2}{|l|}{$a=0$} & \multicolumn{4}{|c|}{$a=0.5$} & \multicolumn{2}{|c|}{$a=1$} \\
\hline & & $\left(1+\frac{1}{\beta}\right) f^{\prime \prime}(0)$ & & $\left(1+\frac{1}{\beta}\right) f^{\prime \prime}($ & & $\left(1+\frac{1}{\beta}\right) g^{\prime \prime}$ & & $\left(1+\frac{1}{\beta}\right) g^{\prime \prime}($ & \\
\hline & & Present & Nadeem et al. (2014) & $\begin{array}{l}\text { Present } \\
\end{array}$ & Nadeem et al. (2014) & Present & Nadeem et al. (2014) & Present & Nadeem et al. (2014) \\
\hline$\infty$ & 0 & 1.00000 & 1.0042 & 1.09310 & 1.0932 & 0.46520 & 0.4653 & 1.17372 & 1.1748 \\
\hline 5 & & 1.09545 & 1.0954 & 1.19743 & 1.1974 & 0.50961 & 0.5096 & 1.28575 & 1.2857 \\
\hline 1 & & 1.41421 & 1.4142 & 1.54587 & 1.5459 & 0.65790 & 0.6579 & 1.65989 & 1.6599 \\
\hline$\infty$ & 10 & 3.31662 & 3.3165 & 3.34204 & 3.3420 & 1.64590 & 1.6459 & 3.36724 & 3.667 \\
\hline 5 & & 3.63318 & 3.6331 & 3.66102 & 3.6610 & 1.80299 & 1.8030 & 3.68863 & 3.6886 \\
\hline 1 & & 4.69042 & 4.6904 & 4.72635 & 4.7263 & 2.32765 & 2.3276 & 4.76200 & 4.7620 \\
\hline$\infty 1$ & 100 & 10.04988 & 10.049 & 10.05818 & 10.058 & 5.02080 & 5.0208 & 10.06648 & 10.066 \\
\hline 5 & & 11.00909 & 11.0091 & 11.01818 & 11.0182 & 5.50001 & 5.5000 & 11.02727 & 11.0272 \\
\hline 1 & & 14.21267 & 14.2127 & 14.22441 & 14.2244 & 7.10048 & 7.1004 & 14.23614 & 14.2361 \\
\hline
\end{tabular}

Casson fluid in the region of stagnation-point towards a stretching sheet with effects of viscous dissipation. Bhattacharyya (2013) considered the two-dimensional magnetohydrodynamic stagnation-point flow and heat transfer of an electrically conducting non-Newtonian Casson fluid towards a stretching sheet in the presence of thermal radiation. Mukhopadhyay et al. (2013) investigated the unsteady two dimensional flow of a non-Newtonian fluid over a stretching surface with a prescribed surface temperature. Nandy (2013) investigated the hydromagnetic boundary layer flow and heat transfer of a non-Newtonian Casson fluid in the neighborhood of a stagnation point over a stretching sheet in the presence of velocity and thermal slip at the boundary. Nadeem et al. (2013) examined magnetohydrodynamic Casson fluid flow in two lateral dimensions past a porous linear stretching sheet. Again, Nadeem et al. (2014) studied the steady flow of a Casson fluid in the presence of nanoparticles. In the work, they considered the fact that the sheet was stretched in the $x y$-plane and formulated a three-dimensional MHD boundary layer flow model for a Casson nanofluid past a linearly stretching sheet with a convective boundary condition. Kunetsov and Nield (2014) revisited their model on natural convective boundary layer flow over a vertical plate by incorporating the effects of Brownian and thermophoretic diffusion coefficients on the nanoparticle concentration at the boundary condition to make the nanoparticle fraction at the boundary passive instead of actively controlled by assuming that the nanoparticle flux at the wall is zero.

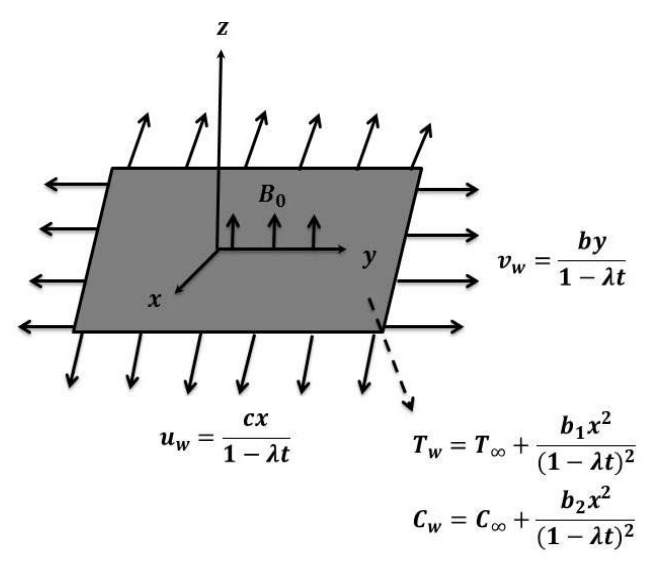

Fig. 1 Schematic flow diagram for the three dimensional problem

In this work, the fluid flow, heat and mass transfer in a Casson fluid containing suspensions of nanoparticles is studied with combined effects of various parameters entering the flow problem. The combined effects of velocity slip with thermal convection condition on unsteady three dimensional magnetohydrodynamic flow of a Casson nanofluid together with an assumption of a zero nanoparticle flux at the concentration boundary has not been studied to the best knowledge of the authors. The problem is formulated for a three dimensional magnetohydrodynamic boundary layer flow of an unsteady Casson nanofluid past a linearly stretching porous sheet with partial slip and convective boundary conditions, respectively with the revised nanofluid model. The aim of this work is to study the effects of thermal radiation, heat generation, porosity and some other parameters discussed in the problem on the unsteady Casson nanofluid model with respect to effects of partial slip, convective and Brownian and thermophoresis diffusion coefficients on the momentum, thermal and nanoparticle concentration boundary conditions respectively. Much emphasis is laid on the nanoparticles concentration boundary conditions which is made to be more realistic due to the incorporation of the Brownian and thermophoresis diffusion coefficients and its effect on the fluid model studied. The partial differential equations of the fluid model are transformed into a system of non-linear ordinary differential equations using the similarity transformation and the non-dimensional governing equations are solved using spectral quasilinearisation method(SQLM). A valid comparison of the obtained solution with existing literature is given and the effect of the dimensionless parameters on the flow, heat and mass transfer of the fluid model is varied and discussed in tabular and graphical forms.

\section{MATHEMATICAL MODEL}

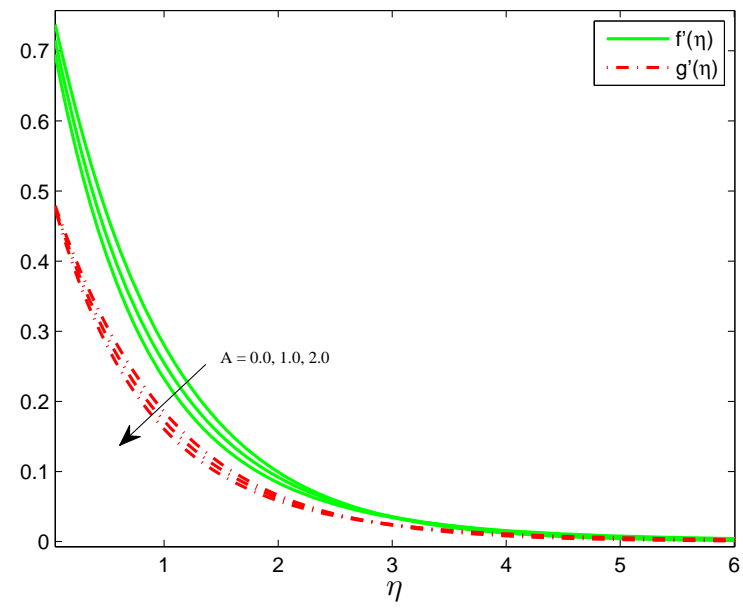

Fig. 2 Effect of the unsteadiness parameter A on the velocity profiles $f^{\prime}(\eta)$ and $g^{\prime}(\eta)$ when $a=0.5, \mathrm{He}=0.3, N b=0.3, N t=0.1, E c=$ $0.1, B i=N_{R}=M=\Lambda=\beta=0.5, \delta=0.1, P r=10$ and $L e=1$.

Consider an unsteady three-dimensional laminar, incompressible Casson nanofluid flow over linearly stretching porous sheet. The unsteady fluid flow starts at $\mathrm{t}=0$ with linear stretching velocities $u=\frac{c x}{1-\lambda t}$ and $v=\frac{b y}{1-\lambda t}$ along the $x, y$-plane respectively, where $b, c$ are positive 


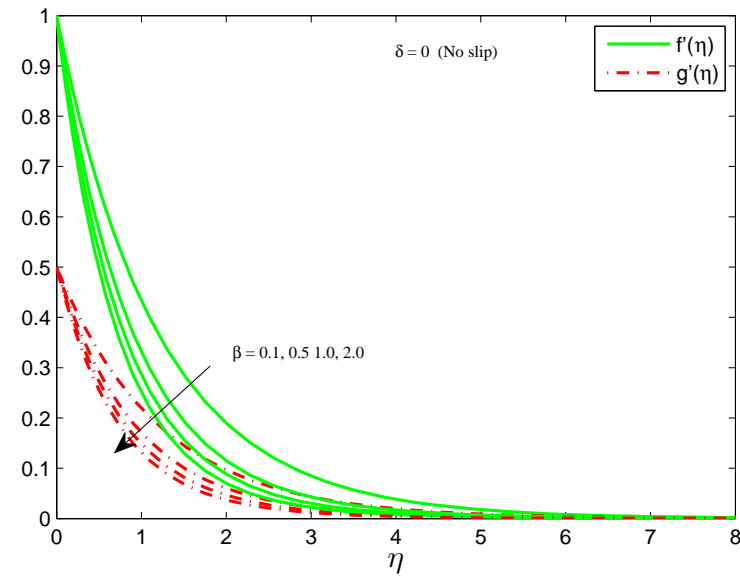

(a) $\delta=0$ No-Slip boundary condition

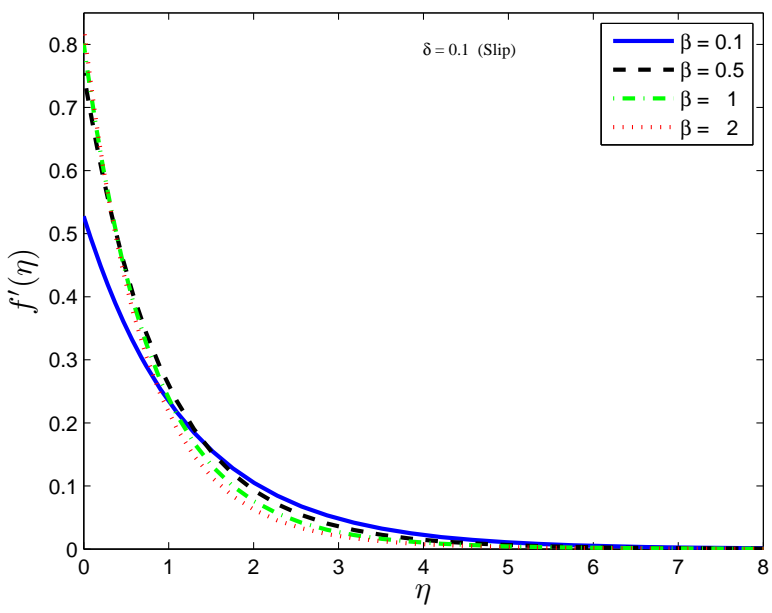

(b) $\delta=0.1$ Navier Slip boundary condition

Fig. 3 Effect of the Casson parameter $\beta$ on the velocity profiles $f^{\prime}(\eta)$ and $g^{\prime}(\eta)$ for (a) No slip and (b) Navier velocity slip boundary condition when $A=0.8, a=0.5, H e=0.3, E c=0.1, M=B i=N_{R}=\Lambda=$ $0.5, N b=0.3, N t=0.1, P r=10$ and $L e=1$.

constants and $\lambda \geq 0$. The fluid is placed along the $z$-axis and a constant magnetic field of strength $B_{0}$ is applied in a direction normal to the surface of the flow as shown in Figure 1. The rheological equation of state for an isotropic and incompressible flow of a Casson fluid is expressed as (Mukhopadhyay et al. (2013)):

$$
\tau_{i j}= \begin{cases}2\left(\mu_{B}+\frac{p_{y}}{\sqrt{2 \pi}}\right) e_{i j} & \text { if } \pi>\pi_{c} \\ 2\left(\mu_{B}+\frac{p_{y}}{\sqrt{2 \pi_{c}}}\right) e_{i j} & \text { if } \pi<\pi_{c}\end{cases}
$$

where $e_{i j}$ is the $(i, j)$ th component of the rate of strain tensor, $\tau_{i j}$ is the $(i, j)$ th component of the stress tensor, $\mu_{B}$ is the Casson coefficient of viscosity, $\pi=e_{i j} e_{i j}$ is the product of the rate of strain tensor with itself, $\pi_{c}$ is the critical value of the product of the rate of strain tensor with itself and $p_{y}$ is the yield stress of the fluid. It is assumed that both the temperature and the concentration at the surface vary with distance and time, respectively from the origin. The temperature $T_{w}$ and the concentration $C_{w}$ at the surface are given by:

$$
T_{w}=T_{\infty}+\frac{b_{1} x^{2}}{(1-\lambda t)^{2}}, \quad C_{w}=C_{\infty}+\frac{b_{2} x^{2}}{(1-\lambda t)^{2}}
$$

where $b_{1}$ and $b_{2}$ are constants. It should be noted that the expressions $u_{w}$, $v_{w}, T_{w}$, and $C_{w}$ are valid only for $t \leq 1 / \lambda$, but not when $\lambda=0$. The boundary layer equations for the three-dimensional incompressible Casson nanofluid are given as (Nadeem et al. (2014); Ibrahim and Makinde (2015); Sulochana et al. (2016)):

$$
\begin{aligned}
& \frac{\partial u}{\partial x}+\frac{\partial v}{\partial y}+\frac{\partial w}{\partial z}=0 \\
& \frac{\partial u}{\partial t}+u \frac{\partial u}{\partial x}+v \frac{\partial u}{\partial y}+w \frac{\partial u}{\partial z}=\nu\left(1+\frac{1}{\beta}\right) \frac{\partial^{2} u}{\partial z^{2}}-\frac{\sigma B_{o}^{2}}{\rho_{f}} u \\
& -\left(1+\frac{1}{\beta}\right) \frac{\nu}{K} u \\
& \frac{\partial v}{\partial t}+u \frac{\partial v}{\partial x}+v \frac{\partial v}{\partial y}+w \frac{\partial v}{\partial z}=\nu\left(1+\frac{1}{\beta}\right) \frac{\partial^{2} v}{\partial z^{2}}-\frac{\sigma B_{o}^{2}}{\rho_{f}} v \\
& -\left(1+\frac{1}{\beta}\right) \frac{\nu}{K} v, \\
& \frac{\partial T}{\partial t}+u \frac{\partial T}{\partial x}+v \frac{\partial T}{\partial y}+w \frac{\partial T}{\partial z}=\frac{k_{T}}{\rho c_{p}} \frac{\partial^{2} T}{\partial z^{2}}+\frac{\nu}{c_{p}}\left(1+\frac{1}{\beta}\right)\left(\frac{\partial u}{\partial z}\right)^{2} \\
& -\frac{1}{\rho c_{p}} \frac{\partial q_{r}}{\partial z}+\frac{Q}{\rho c_{p}}\left(T-T_{\infty}\right)+\tau\left[D_{B} \frac{\partial C}{\partial z} \frac{\partial T}{\partial z}+\frac{D_{T}}{T_{\infty}}\left(\frac{\partial T}{\partial z}\right)^{2}\right] \\
& \frac{\partial C}{\partial t}+u \frac{\partial C}{\partial x}+v \frac{\partial C}{\partial y}+w \frac{\partial C}{\partial z}=D_{B} \frac{\partial^{2} C}{\partial z^{2}}+\frac{D_{T}}{T_{\infty}} \frac{\partial^{2} T}{\partial z^{2}}
\end{aligned}
$$

where $u, v$ and $w$ are the velocities in the $x$-, $y$ - and $z$-directions respec-

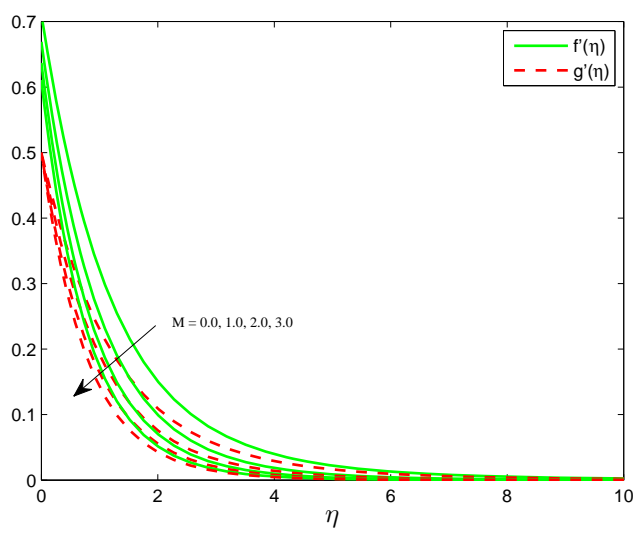

Fig. 4 Effect of the Magnetic field parameter $\mathrm{M}$ on the velocity profiles $f^{\prime}(\eta)$ and $g^{\prime}(\eta)$ when $A=0.8, a=0.5, H e=0.3, E c=0.1, B i=N_{R}=$ $\Lambda=\beta=0.5, \delta=0.1, N b=0.3, N t=0.1, P r=10$ and $L e=1$.

tively, $\beta=\mu_{B} \frac{\sqrt{2 \pi_{c}}}{p_{y}}$ is the Casson fluid parameter, $\nu$ is the kinematic viscosity, $B_{o}$ is the magnetic induction, $K$ is the porous medium permeability, $\sigma$ is the electrical conductivity, $k_{T}$ is the thermal diffusivity, $q_{r}=-\frac{4 \sigma^{*}}{3 K^{*}} \frac{\partial T^{4}}{\partial z}$ is the radiation heat flux, $Q_{o}$ is the heat generation constant, $\tau=\frac{(\rho c)_{p}}{(\rho c)_{f}}$ is the ratio of the heat capacity of the nanoparticle material and the heat capacity of the fluid, $D_{B}$ is the Brownian diffusion coefficient, $D_{T}$ is the thermophoretic diffusion coefficient. The associated boundary conditions are;

$$
\begin{aligned}
& u=u_{w}+u_{\text {slip }}, v=v_{w}, w=0,-k_{f}\left(\frac{\partial T}{\partial z}\right)=h_{f}\left(T_{w}-T\right), \\
& D_{B} \frac{\partial C}{\partial z}+\frac{D_{T}}{T_{\infty}} \frac{\partial T}{\partial z}=0, \quad \text { at } \quad z=0 \\
& u \rightarrow 0, \quad v \rightarrow 0, \quad T \rightarrow T_{\infty}, \quad C \rightarrow C_{\infty}, \quad \text { as } \quad z \rightarrow \infty
\end{aligned}
$$


Here,

$$
\begin{aligned}
& u_{w}=\frac{c x}{1-\lambda t}, u_{\text {slip }}=L\left(\mu_{B}+\frac{p_{y}}{\sqrt{2 \pi_{c}}}\right) \frac{\partial u}{\partial z}, \\
& L=N(1-\lambda t)^{\frac{1}{2}} \text { and } v_{w}=\frac{b y}{1-\lambda t}
\end{aligned}
$$

where $b$ and $c$ are positive constants, $u_{w}$ and $v_{w}$ are stretching velocities in the $x$ - and $y$-directions respectively, $L$ is the velocity slip factor, $T_{w}$ is the temperature at the wall, $C_{w}$ is the concentration at the wall, the constant values $T_{\infty}$ and $C_{\infty}$ are the temperature and concentration far away from the wall respectively, $k_{f}$ is the thermal conductivity of the fluid and $h_{f}$ is the convective heat transfer coefficient. Introducing the following similarity transformations(see Mukhopadhyay et al. (2013); Mukhopadhyay (2013a)):

$$
\begin{aligned}
& u=\frac{c x f^{\prime}(\eta)}{1-\lambda t}, v=\frac{c y g^{\prime}(\eta)}{1-\lambda t}, w=-\sqrt{\frac{c \nu}{1-\lambda t}}(f(\eta)+g(\eta)), \\
& a=b / c, \eta=z \sqrt{\frac{c}{\nu(1-\lambda t)}}, T_{w}=T_{\infty}+\frac{b_{1} x^{2}}{(1-\lambda t)^{2}} \theta(\eta), \\
& C_{w}=C_{\infty}+\frac{b_{2} x^{2}}{(1-\lambda t)^{2}} \phi(\eta)
\end{aligned}
$$

where $a=\frac{b}{c}$ is the ratio of the velocities in $y$ - and $x$-directions, and the prime denotes differentiation with respect to $\eta$. Using Eq. (11), continuity is easily satisfied and Eqs. (4)-(7) together with the boundary conditions (8) and (9), lead to the non-dimensional equations;

$$
\begin{aligned}
& \left(1+\frac{1}{\beta}\right) f^{\prime \prime \prime}-A\left(f^{\prime}+\frac{\eta}{2} f^{\prime \prime}\right)-f^{2}+(f+g) f^{\prime \prime} \\
& -\left(M+\left(1+\frac{1}{\beta}\right) \Lambda\right) f^{\prime}=0, \\
& \left(1+\frac{1}{\beta}\right) g^{\prime \prime \prime}-A\left(g^{\prime}+\frac{\eta}{2} g^{\prime \prime}\right)-g^{2}+(f+g) g^{\prime \prime} \\
& -\left(M+\left(1+\frac{1}{\beta}\right) \Lambda\right) g^{\prime}=0, \\
& \frac{1}{\operatorname{Pr}}\left(1+N_{R}\right) \theta^{\prime \prime}-A\left(2 \theta+\frac{\eta}{2} \theta^{\prime}\right)-2 f^{\prime} \theta+(f+g) \theta^{\prime} \\
& +\left(1+\frac{1}{\beta}\right) E c f^{\prime \prime 2}+N b \theta^{\prime} \phi^{\prime}+N t \theta^{2}+H e \theta=0, \\
& \phi^{\prime \prime}-A \operatorname{Pr} L e\left(2 \phi+\frac{\eta}{2} \phi^{\prime}\right)-2 \operatorname{Pr} L e f^{\prime} \phi+\operatorname{PrLe}(f+g) \phi^{\prime} \\
& +\frac{N t}{N b} \theta^{\prime \prime}=0,
\end{aligned}
$$

subject to the boundary conditions

$$
\begin{aligned}
& f(0)=0, f^{\prime}(0)=1+\delta\left(1+\frac{1}{\beta}\right) f^{\prime \prime}(0), g(0)=0, \\
& g^{\prime}(0)=a, f^{\prime}(\infty)=0, g^{\prime}(\infty)=0 \\
& \theta^{\prime}(0)=-B i(1-\theta(0)), N b \phi^{\prime}(0)+N t \theta^{\prime}(0)=0 \\
& \theta(\infty)=0, \phi(\infty)=0
\end{aligned}
$$

where $f(\eta), \theta(\eta)$ and $\phi(\eta)$ are the velocity, temperature and concentration respectively. The dimensionless parameters are; $A=\frac{\lambda}{c}$ is the unsteadiness parameter, $M=\frac{\sigma B_{0}^{2}}{c \rho}$ is the magnetic parameter, $\Lambda=$ $\frac{\nu(1-\lambda t)}{K c}$ is the porosity parameter, $\operatorname{Pr}=\frac{\nu \rho c_{p}}{k_{T}}$ is the Prandtl number, $H e=\frac{Q_{0}}{c \rho c_{p}}$ is the heat generation parameter, $N b=\frac{\tau D_{B}\left(C_{w}-C_{\infty}\right)}{\nu}$ is the Brownian motion parameter, $N t=\frac{\tau D_{T}\left(T_{w}-T_{\infty}\right)}{\nu T_{\infty}}$ is the thermophoresis parameter, $E c=\frac{c^{2}}{b_{1} c_{p}}$ is the Eckert number, $N_{R}=\frac{16 \sigma^{*} T_{\infty}^{3}}{3 k^{*} k_{T}}$ is the radiation parameter, $L e=\frac{k_{T}}{D_{B} \rho c_{p}}$ is the Lewis number, $\delta=$ $N \mu_{B} \sqrt{\frac{c}{\nu}}$ is the dimensionless velocity slip parameter, $B i=\frac{h_{f}}{k_{0}} \sqrt{\frac{\nu}{c}}$ is the Biot number.

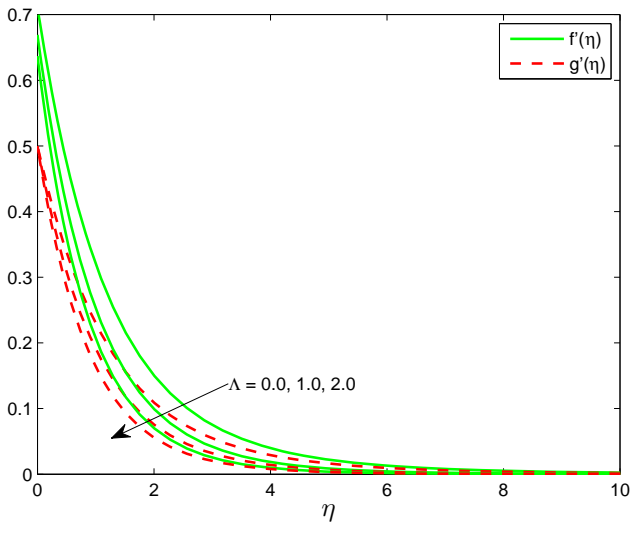

Fig. 5 Effect of the Porosity parameter $\Lambda$ on the velocity profiles $f^{\prime}(\eta)$ and $g^{\prime}(\eta)$ when $A=0.8, a=0.5, H e=0.3, E c=0.1, M=B i=N_{R}=$ $\beta=0.5, N b=0.3, N t=0.1, \delta=0.1, \operatorname{Pr}=10$ and $L e=1$.

\section{SKIN FRICTION, RATE OF HEAT TRANSFER}

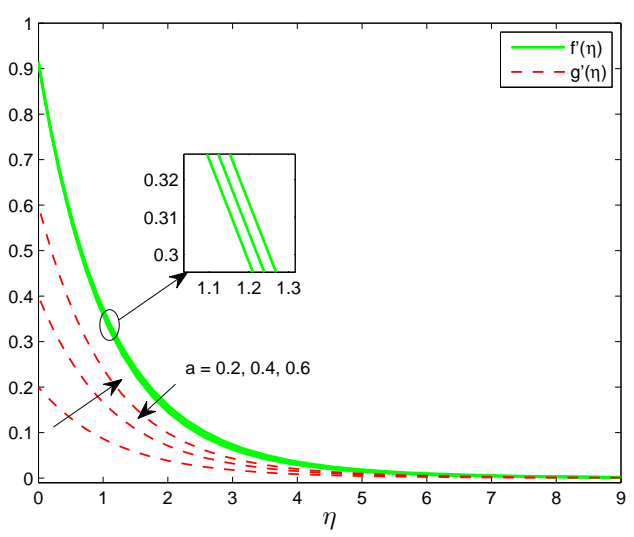

Fig. 6 Effect of the Stretching ratio parameter a on the velocity profiles $f^{\prime}(\eta)$ and $g^{\prime}(\eta)$ when $A=0.8, \mathrm{He}=0.3, E c=0.1, M=B i=N_{R}=\Lambda=$ $\beta=0.5, \delta=0.1, N b=0.3, N t=0.1, \operatorname{Pr}=10$ and $L e=1$.

For the physical quantities of engineering primary interests, the local wall shear stresses along the $\mathrm{x}$ - and $\mathrm{y}$-directions, denoted by $\tau_{w x}$ and $\tau_{w y}$ respectively and local surface heat flux $q_{w}$ are evaluated respectively from the following definitions:

$$
\begin{aligned}
& \tau_{w x}=\left(\mu_{B}+\frac{p_{y}}{\sqrt{2 \pi_{c}}}\right)\left(\frac{\partial u}{\partial z}\right)_{z=0}, \\
& \tau_{w y}=\left(\mu_{B}+\frac{p_{y}}{\sqrt{2 \pi_{c}}}\right)\left(\frac{\partial v}{\partial z}\right)_{z=0}, \quad q_{w}=-k\left(\frac{\partial T}{\partial z}\right)_{z=0} .
\end{aligned}
$$

The dimensionless skin friction coefficient $C_{f}$ on the surface along the x and y directions denoted by $C_{f x}$ and $C_{f y}$, respectively and the local Nusselt number $N u_{x}$ are defined as:

$$
C_{f x}=\frac{\tau_{w x}}{\rho u_{w}^{2}}, \quad C_{f y}=\frac{\tau_{w y}}{\rho u_{w}^{2}}, \quad N u_{x}=\frac{x q_{w}}{k\left(T_{w}-T_{\infty}\right)},
$$

Hence using the similarity variables given in 11 , we obtain

$$
\begin{aligned}
& \operatorname{Re}_{x}^{\frac{1}{2}} C_{f x}=\left(1+\frac{1}{\beta}\right) f^{\prime \prime}(0), \\
& \left(\frac{x}{y}\right) \operatorname{Re}_{x}^{\frac{1}{2}} C_{f y}=a\left(1+\frac{1}{\beta}\right) g^{\prime \prime}(0), R e_{x}^{-\frac{1}{2}} N u_{x}=-\theta^{\prime}(0),
\end{aligned}
$$

where $R e_{x}=\frac{x u_{w}(x)}{\nu}$ is the local Reynolds number. 


\section{NUMERICAL SOLUTION}

The ordinary differential equations presented in Eqs. (12 - 15) are solved numerically using the Spectral Quasi-linearisation method (SQLM). The SQLM is a numerical technique in which the governing nonlinear equations are linearised using the Newton-Raphson based quasilinearization method (QLM) which was first developed by Bellman and Kalaba (1965), then integrated using Chebyshev spectral collocation method. Some of the characteristics of the method are the fact that it is efficient in terms of computational accuracy and exhibits fast convergence (Motsa et al. (2014a); Motsa et al. (2014b)). The QLM technique uses the ideas of Newton-Rapson method to linearise nonlinear ordinary differential equations (ODEs) or partial differential equations (PDEs) and the scheme is derived by linearising the nonlinear components of the governing equations using Taylor series expansion with the assumption that the difference between the value of the unknown function at the current iteration level denoted by $r+1$ and the value of the previous iteration level denoted by $r$ is small. For a detailed description of the method, see Motsa (2013).

\section{Method Description}

The four system of nonlinear ordinary differential equations in four unknowns $(f(\eta), g(\eta), \theta(\eta)$ and $\phi(\eta))$ given in Eqs. (12 - 15), are written as a sum of its linear and nonlinear components and the nonlinear components linearised using one term Taylor series for multiple variables to give the following:

$$
\begin{aligned}
& a_{0, r} f_{r+1}^{\prime \prime \prime}+a_{1, r} f_{r+1}^{\prime \prime}+a_{2, r} f_{r+1}^{\prime}+a_{3, r} f_{r+1} \\
& +a_{4, r} g_{r+1}=R_{1}, \\
& \quad b_{0, r} g_{r+1}^{\prime \prime \prime}+b_{1, r} g_{r+1}^{\prime \prime}+b_{2, r} g_{r+1}^{\prime}+b_{3, r} g_{r+1} \\
& +b_{4, r} f_{r+1}=R_{2}, \\
& \quad c_{0, r} \theta_{r+1}^{\prime \prime}+c_{1, r} \theta_{r+1}^{\prime}+c_{2, r} \theta_{r+1}+c_{3, r} f_{r+1}^{\prime \prime}+c_{4, r} f_{r+1}^{\prime} \\
& +c_{5, r} f_{r+1}+c_{6, r} g_{r+1}+c_{7, r} \phi_{r+1}^{\prime}=R_{3}, \\
& \quad \phi_{r+1}^{\prime \prime}+d_{1, r} \phi_{r+1}^{\prime}+d_{2, r} \phi_{r+1}+d_{3, r} f_{r+1}^{\prime}+d_{4, r} f_{r+1} \\
& +d_{5, r} g_{r+1}+d_{6, r} \theta_{r+1}^{\prime \prime}=R_{4},
\end{aligned}
$$

where $a_{i, r}, b_{i, r}, c_{i, r}$ and $d_{i, r}(i=1,2,3, \ldots)$ are known from previous calculations and are given by:

$$
\begin{aligned}
& a_{0, r}=1+\frac{1}{\beta}, a_{1, r}=f_{r}-A \frac{\eta}{2}+g_{r}, a_{2, r}=-A-2 f_{r}^{\prime}-M \\
& -\left(1+\frac{1}{\beta}\right) \Lambda, a_{3, r}=a_{4, r}=f_{r}^{\prime \prime}, \\
& b_{0, r}=1+\frac{1}{\beta}, b_{1, r}=f_{r}-A \frac{\eta}{2}+g_{r}, b_{2, r}=-A-2 g_{r}^{\prime}-M \\
& -\left(1+\frac{1}{\beta}\right) \Lambda, b_{3, r}=b_{4, r}=g_{r}^{\prime \prime}, \\
& c_{0, r}=\frac{1}{\operatorname{Pr}}\left(1+N_{R}\right), c_{1, r}=f_{r}-A \frac{\eta}{2}+g_{r}+N b \phi_{r}^{\prime}+2 N t \theta_{r}^{\prime}, \\
& c_{2, r}=H e-2 A-2 f_{r}^{\prime}, c_{3, r}=2\left(1+\frac{1}{\beta}\right) \operatorname{Ec} f_{r}^{\prime \prime}, c_{4, r}=-2 \theta_{r}, \\
& c_{5, r}=c_{6, r}=\theta_{r}^{\prime}, c_{7, r}=N b \theta_{r}^{\prime}, \\
& d_{1, r}=\operatorname{Pr} \operatorname{Le}\left(f_{r}-A_{\frac{\eta}{2}}+g_{r}\right), d_{2, r}=-2 \operatorname{Pr} \operatorname{Le}\left(A+f_{r}^{\prime}\right), \\
& d_{3, r}=-2 \operatorname{Pr} \operatorname{Le} \phi_{r}, d_{4, r}=d_{5, r}=\operatorname{Pr} \operatorname{Le} \phi_{r}^{\prime}, \quad d_{6, r}=\frac{N t}{N b}
\end{aligned}
$$

and the right hand side is given as:

$$
\begin{aligned}
& R_{1}=f_{r}^{\prime \prime} f_{r}-f_{r}^{\prime 2}+f_{r}^{\prime \prime} g_{r}, \quad R_{2}=g_{r}^{\prime \prime} g_{r}-g_{r}^{\prime 2}+f_{r} g_{r}^{\prime \prime}, \\
& R_{3}=f_{r} \theta_{r}^{\prime}-2 f_{r}^{\prime} \theta_{r}+g_{r} \theta_{r}^{\prime}+N b \theta_{r}^{\prime} \phi_{r}^{\prime}+N t \theta_{r}^{\prime 2}+\left(1+\frac{1}{\beta}\right) E c f_{r}^{\prime \prime 2}, \\
& R_{4}=\operatorname{Pr} L e f_{r} \phi_{r}^{\prime}-2 \operatorname{Pr} L e f_{r}^{\prime} \phi_{r}+\operatorname{Pr} \operatorname{Leg}_{r} \phi_{r}^{\prime} .
\end{aligned}
$$

Equations (21 - 24) form the iterative scheme for the spectral quasilinearisation method and it is then solved using the Chebyshev spectral collocation method. Starting with suitable initial approximations, the iteration schemes (21) - (24) are then solved iteratively for $f_{r+1}, g_{r+1}, \theta_{r+1}$, and $\phi_{r+1}$ when $r=0,1,2, \ldots$

In solving (21) - (24), the equations are discretised using the Chebyshev spectral collocation method which is implemented on the interval $[-1,1]$. Thus, for convenience of the numerical computations, before applying the spectral method, it is necessary to transform the semi-infinite domain of the problem to an approximated truncated domain $[0, L]$ where $L$ is a finite number selected to be large enough to represent the behavior of the flow properties when $\eta$ is very large. The transformation $\eta=\frac{1}{2} L(\xi+1)$ is used to transform the interval $[0, L]$ to $[-1,1]$. We introduce the differentiation matrix $\mathbf{D}$ which is used to estimate the derivatives of the unknown variables $f(\eta), g(\eta), \theta(\eta)$ and $\phi(\eta)$ at the collocation points as a matrix vector product, that is:

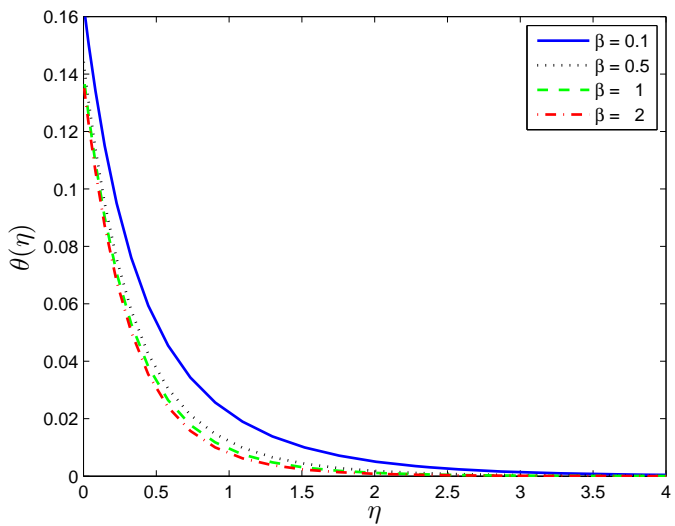

Fig. 7 Effect of the Casson parameter $\beta$ on the temperature profile $\theta(\eta)$ when $A=0.8, a=0.5, \mathrm{He}=0.3, E c=0.1, M=B i=N_{R}=\Lambda=$ $0.5, \delta=0.1, N b=0.3, N t=0.1, P r=10$ and $L e=1$.

$$
\frac{d f}{d \eta}=\sum_{j=0}^{N} \mathbf{D}_{j k} f\left(\xi_{j}\right)=\mathbf{D} F, \quad k=1,2, \ldots, N
$$

where $N+1$ is the number of collocation points, $\mathbf{D}=2 D / L$ and $F=\left[f\left(\xi_{0}\right), f\left(\xi_{1}\right), \ldots, f\left(\xi_{N}\right)\right]^{T}$ is a vector function at the collocation points. Higher order derivatives are obtained as powers of $\mathbf{D}$, that is

$$
F^{s}(\eta)=\mathbf{D}^{s} F,
$$

where $s$ is the order of the derivative and matrix $\mathbf{D}$ is of size $(N+1) \times$ $(N+1)$. The Guass-Lobatto points are chosen to define the nodes in $[-1,1]$ as

$$
\xi_{i}=\cos \frac{\pi i}{N}, \quad i=0,1, \ldots, N ; \quad-1 \leq \xi \leq 1 .
$$

Applying the spectral method to the scheme given in equations (21 - 24) and expressing it in matrix form, we have:

$$
\left[\begin{array}{llll}
A_{11} & A_{12} & A_{13} & A_{14} \\
A_{21} & A_{22} & A_{23} & A_{24} \\
A_{31} & A_{32} & A_{33} & A_{34} \\
A_{41} & A_{42} & A_{43} & A_{44}
\end{array}\right]\left[\begin{array}{l}
F_{r+1} \\
G_{r+1} \\
\Theta_{r+1} \\
\Phi_{r+1}
\end{array}\right]=\left[\begin{array}{l}
R_{1, r} \\
R_{2, r} \\
R_{3, r} \\
R_{4, r}
\end{array}\right],
$$


where $A_{i j}$ and $R_{i, r}(i, j=1,2,3,4)$ are $(N+1) \times(N+1)$ matrices and $(N+1) \times 1$ vectors respectively, defined as:

$$
\begin{aligned}
& A_{11}=a_{0, r} \mathbf{D}^{3}+\left[a_{1, r}\right]_{d} \mathbf{D}^{2}+\left[a_{2, r}\right]_{d} \mathbf{D}+\left[a_{3, r}\right]_{d} \mathbf{I}, \\
& A_{12}=\left[a_{4, r}\right]_{d} \mathbf{I}, \quad A_{13}=\mathbf{O}, \quad A_{14}=\mathbf{O}, \\
& A_{21}=\left[b_{4, r}\right]_{d} \mathbf{I}, \quad A_{22}=b_{0, r} \mathbf{D}^{3}+\left[b_{1, r}\right]_{d} \mathbf{D}^{2}+\left[b_{2, r}\right]_{d} \mathbf{D} \\
& +\left[b_{3, r}\right]_{d} \mathbf{I}, \quad A_{23}=\mathbf{O}, \quad A_{24}=\mathbf{O}, \\
& A_{31}=\left[c_{3, r}\right]_{d} \mathbf{D}^{2}+\left[c_{4, r}\right]_{d} \mathbf{D}+\left[c_{5, r}\right]_{d} \mathbf{I}, \quad A_{32}=\left[c_{6, r}\right]_{d} \mathbf{I}, \\
& A_{33}=c_{0, r} \mathbf{D}^{2}+\left[c_{1, r}\right]_{d} \mathbf{D}+\left[c_{2, r}\right]_{d} \mathbf{I}, \quad A_{34}=\left[c_{7, r}\right]_{d} \mathbf{D}, \\
& A_{41}=\left[d_{3, r}\right]_{d} \mathbf{D}+\left[d_{4, r}\right]_{d} \mathbf{I}, \quad A_{42}=\left[d_{5, r}\right]_{d} \mathbf{I}, \\
& A_{43}=d_{6, r} \mathbf{D}^{2}, \quad A_{44}=\mathbf{D}^{2}+\left[d_{1, r}\right]_{d} \mathbf{D}+\left[d_{2, r}\right]_{d} \mathbf{I} .
\end{aligned}
$$

and $[\cdots]_{d}$ denotes diagonal matrix.

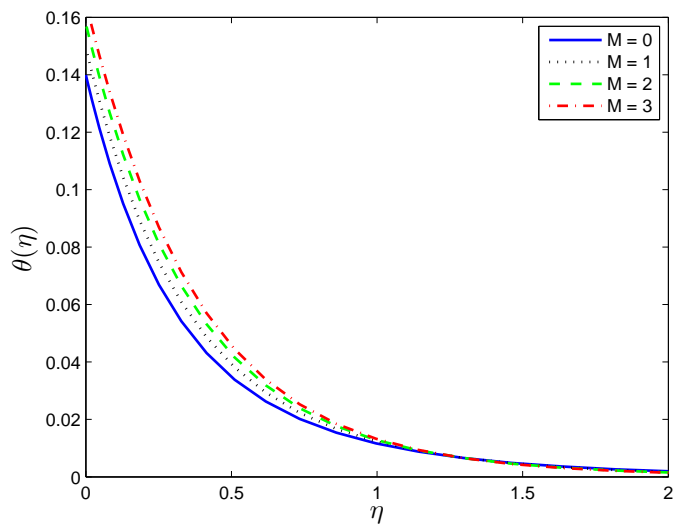

Fig. 8 Effect of the Magnetic field parameter $\mathrm{M}$ on the temperature profile $\theta(\eta)$ when $A=0.8, a=0.5, H e=0.3, E c=0.1, B i=N_{R}=\Lambda=\beta=$ $0.5, \delta=0.1, N b=0.3, N t=0.1, P r=10$ and $L e=1$.

\section{RESULTS AND DISCUSSION}

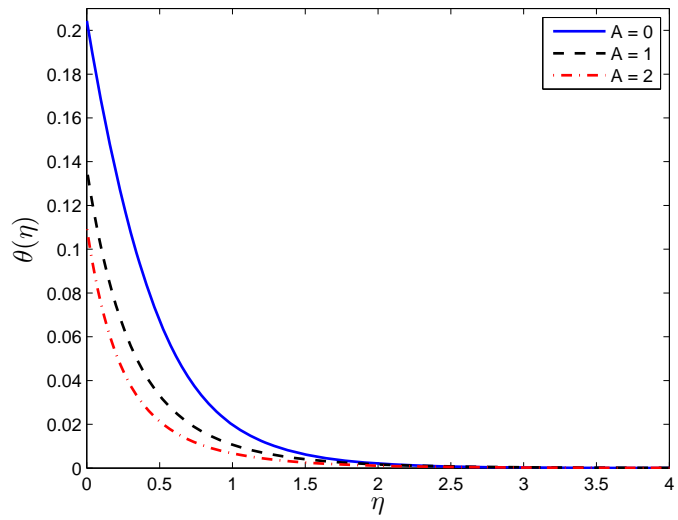

Fig. 9 Effect of the unsteadiness parameter $\mathrm{A}$ on the temperature profile $\theta(\eta)$ when $a=0.5, H e=0.3, N b=0.3, N t=0.1, E c=0.1, B i=$ $N_{R}=M=\Lambda=\beta=0.5, \delta=0.1, \operatorname{Pr}=10$ and $L e=1$.

In this work, we studied partial slip and convective condition imposed on the velocity and thermal boundary conditions respectively with an assumption of a zero nanoparticle flux on the concentration boundary condition for boundary layer flow of a three dimensional magnetohydrodynamic Casson nanofluid over an unsteady stretching sheet. The fluid model was studied using the Spectral quasi-linearisation method and a

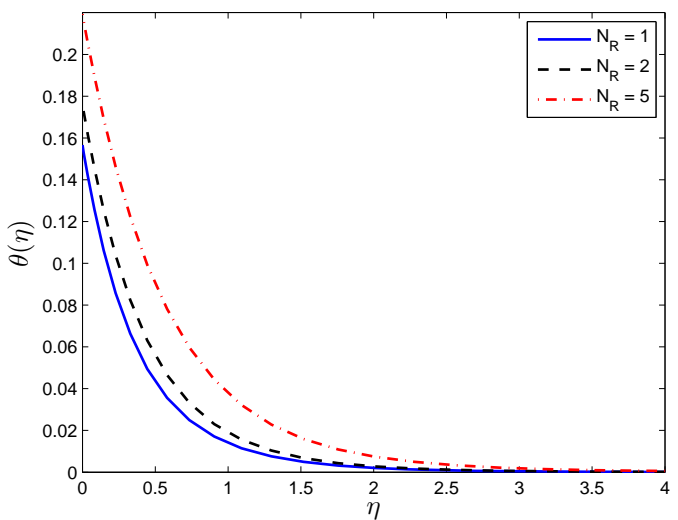

Fig. 10 Effect of the Thermal radiation parameter $N_{R}$ on the temperature profile $\theta(\eta)$ when $A=0.8, a=0.5, H e=0.3, E c=0.1, M=B i=\Lambda=$ $\beta=0.5, \delta=0.1, N b=0.3, N t=0.1, \operatorname{Pr}=10$ and $L e=1$.

comparision of results with previously published literature is provided for validation of results in the present study. It is noteworthy here that we have assumed a constant prandtl number of $\operatorname{Pr}=10$ due to the fact that we are considering a casson nanofluid model for a realistic fluid flow properties.

Table 1 shows a comparison of the skin friction coefficient when varying the stretching ratio $a$ with the results given in Nadeem et al. (2014) when the porosity parameter is zero. We note that a good agreement was achieved between the two set of results.

Figure 2 shows the effect of the unsteadiness parameter on the velocity profiles $f^{\prime}(\eta)$ and $g^{\prime}(\eta)$. It is observed that increasing the unsteady parameter reduces the velocity profiles and this effect is accompanied by a reduction in the momentum boundary layer thickness in both profiles which indicates that the unsteadiness parameter reduces the flowrate due to the stretching sheet.

Figures 3(a) and 3(b) shows the effect of the Casson parameter on the velocity profiles $f^{\prime}(\eta)$ and $g^{\prime}(\eta)$. Due to the Navier velocity slip condition, two scenarios are observed in the figures. The slip boundary condition leads to a transition from non-Newtonian fluid regime to Newtonian, and the physical explanation for this observation is explained viz: It is a known fact that increasing the Casson parameter decreases the yield stress of the Casson fluid, and increasing it indefinitely will make the fluid behave as a Newtonian fluid. It is evident that fluid motion is slowed down in both direction due to increase in the value of the Casson parameter in 3(a) for no-slip boundary condition, which means decrease in the velocity profiles and leads to a decrease in the momentum boundary layer thickness. This behavior is also observed in Nadeem et al. (2014) and Mukhopadhyay (2013a). Additionally, in 3(b), the slip condition has been taken into consideration and we note an increase in the velocity profile $f^{\prime}(\eta)$ close to the wall. Far away from the wall, the fluid tends to behave as a Newtonian fluid as the parameter increases. The velocity slip parameter on the velocity profile $g^{\prime}(\eta)$ remains unchanged with the slip condition and it was not displayed due to this reason.

Figure 4 depicts the effect of the magnetic field parameter on the velocity profiles $f^{\prime}(\eta)$ and $g^{\prime}(\eta)$. It is perceived that increase in the value of the magnetic field parameter leads to a decrease in the velocity profiles. Reduction in the fluid velocity occurs due to the dual interaction of magnetic and electrical forces in the electrically conducting fluid and the applied magnetic field then causes an opposing force called the Lorentz force to the motion of the fluid which in turn leads to a decrease in the momentum boundary layer.

Figure 5 displays the effect of the porosity parameter on the velocity profiles $f^{\prime}(\eta)$ and $g^{\prime}(\eta)$. The porosity parameter tends to oppose flow and then leads to a restriction of fluid flow, thus the speed of the motion 


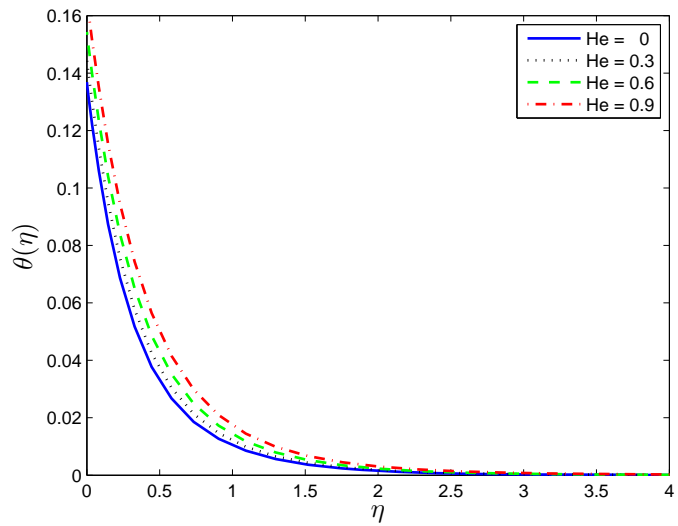

Fig. 11 Effect of the Heat generation parameter He on the temperature profile $\theta(\eta)$ when $A=0.8, a=0.5, E c=0.1, M=B i=N_{R}=\Lambda=\beta=$ $0.5, \delta=0.1, N b=0.3, N t=0.1, \operatorname{Pr}=10$ and $L e=1$.

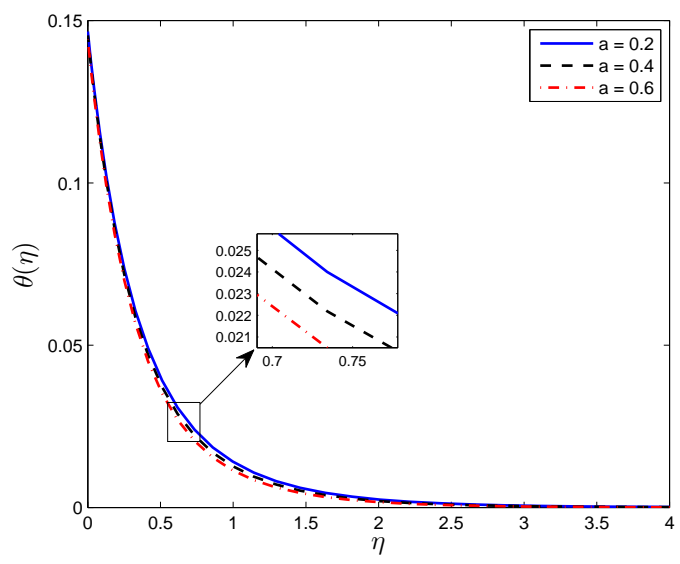

Fig. 12 Effect of the stretching ratio parameter a on the temperature profile $\theta(\eta)$ when $A=0.8, H e=0.3, E c=0.1, M=B i=N_{R}=\Lambda=\beta=$ $0.5, \delta=0.1, N b=0.3, N t=0.1, \operatorname{Pr}=10$ and $L e=1$.

of the fluid is reduced. This flow attribute is noticed from the figure, since the velocity profiles decrease with increase in the values of the porous parameter. We observe that the velocity profiles decrease with the increasing the values of the porous parameter. This in turn leads to a decrease in the velocity boundary layer thickness in both directions.

Figure 6 shows the effect of varying the stretching ratio parameter on the velocity profiles $f^{\prime}(\eta)$ and $g^{\prime}(\eta)$. It is seen that increasing the values of the stretching ratio parameter reduces the velocity profile $f^{\prime}(\eta)$ while it enhances the velocity profile $g^{\prime}(\eta)$. From this parameter, three scenarios are observed: The first is when $a=0$, the flow reduces to two dimensional on the velocity profile $f^{\prime}(\eta)$ only, while for $a=1$, both the velocity profiles $f^{\prime}(\eta)$ and $g^{\prime}(\eta)$ behave the same and have the same solution, a phenomena for axisymmetric flows. In the third scenario, when $0 \leq a \leq 1$, the behavior is shown in Figure 6 and Table 1 .

Figure 7 depicts the effect of the Casson parameter on the temperature profile $\theta(\eta)$. It is seen that an increase in the Casson parameter results in a decrease in the temperature profile. This means suppressing the yield stress while increasing the Casson parameter has a decreasing effect on the thermal boundary layer thickness. This flow pattern is observed in Bhattacharyya (2013) in the case of lower values of the velocity ratio parameter.

Figure 8 shows the effect of the magnetic field parameter on the temperature profile $\theta(\eta)$. It is shown that increasing the magnetic field parameter leads to an increase in the temperature profile since the essence of

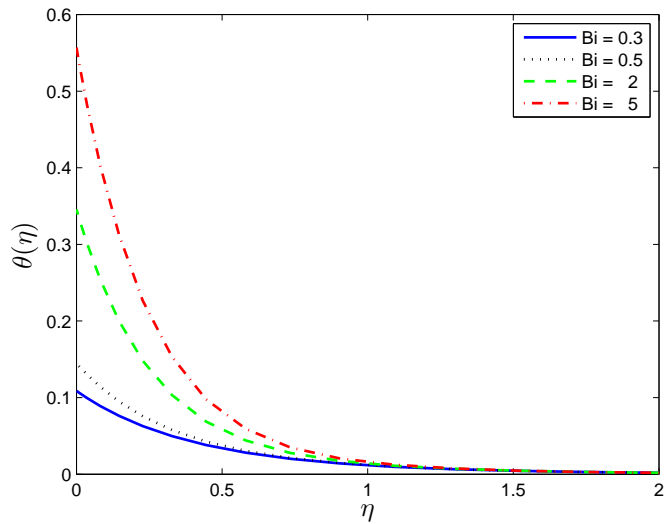

Fig. 13 Effect of the Biot number a on the temperature profile $\theta(\eta)$ when $A=$ $0.8, a=0.5, \mathrm{He}=0.3, E c=0.1, M=N_{R}=\Lambda=\beta=0.5, \delta=$ $0.1, N b=0.3, N t=0.1, P r=10$ and $L e=1$.

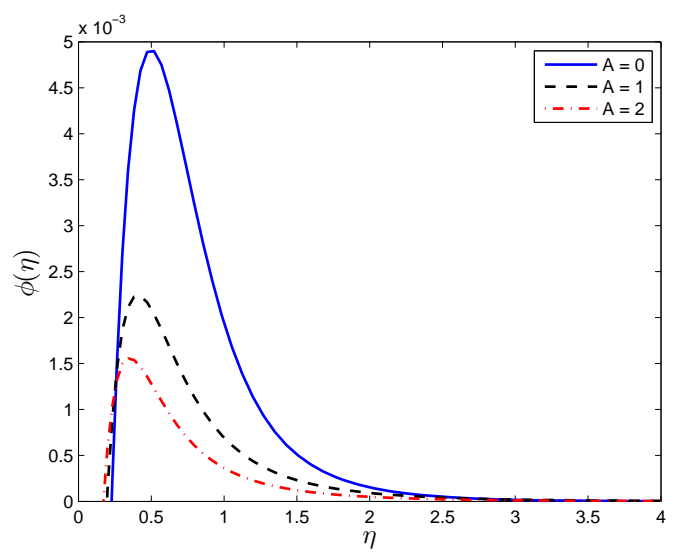

Fig. 14 Effect of the Unsteadiness parameter A on the concentration profile $\phi(\eta)$ when $a=0.5, H e=0.3, N b=0.3, N t=0.1, E c=0.1, B i=$ $N_{R}=M=\Lambda=\beta=0.5, \delta=0.1, \operatorname{Pr}=10$ and $L e=1$

the magnetic field parameter is to enhance the temperature profile of the flow which leads to an increase in the thermal boundary layer thickness as observed in Bhattacharyya (2013).

Figure 9 displays the effect of the unsteadiness parameter on the temperature profile $\theta(\eta)$ and it is observed that the temperature profiles decrease significantly as the unsteady parameter is increased. This is physically so because increasing the unsteadiness enhances heat loss due to the stretching of the sheet, thus resulting in a decrease in the temperature profile. This implies that the rate of cooling is much faster compared to rate of cooling for the steady flow because of the decrease in the rate of heat transfer from the sheet to the fluid for higher values of the unsteady parameter. Similar behavior can be found in Mukhopadhyay et al. (2013).

Figure 10 depicts the effect of the thermal radiation parameter on the temperature profile $\theta(\eta)$. It is seen that increase in the value of thermal radiation parameter enhances the heat transfer which leads to an increase in the thermal boundary layer thickness.

The effect of the heat generation parameter on the temperature profile $\theta(\eta)$ is shown in Figure 11. Heat generation parameter has the characteristic of releasing heat energy to fluid flow for enhancement of the temperature profile and this is as seen from the figure that increase in the Heat generation parameter leads to an increase in the temperature profile, thereby increasing the thermal boundary layer thickness.

The effect of the stretching ratio parameter on the temperature profile $\theta(\eta)$ is shown in Figure 12. It is seen that increasing value the stretching ratio parameter decreases the temperature profile.The fluid temperature 


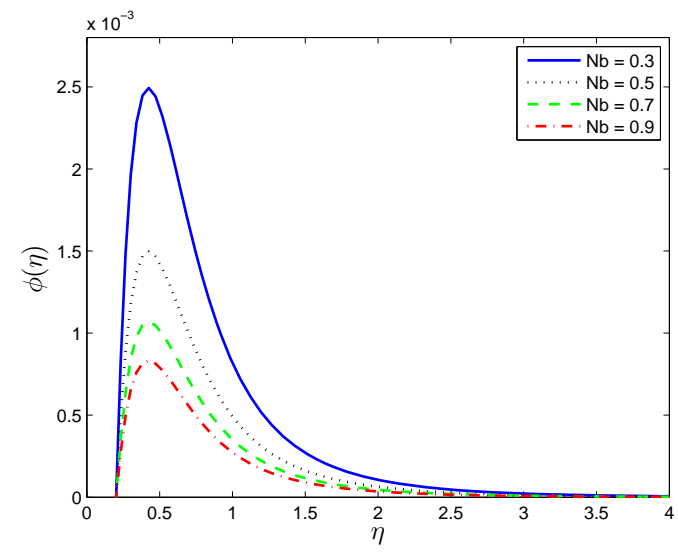

Fig. 15 Effect of the Brownian motion parameter $\mathrm{Nb}$ on the concentration profile $\phi(\eta)$ when $A=0.8, a=0.5, H e=0.3, E c=0.1, M=B i=$ $N_{R}=\Lambda=\beta=0.5, \delta=0.1, N t=0.1, \operatorname{Pr}=10$ and $L e=1$.

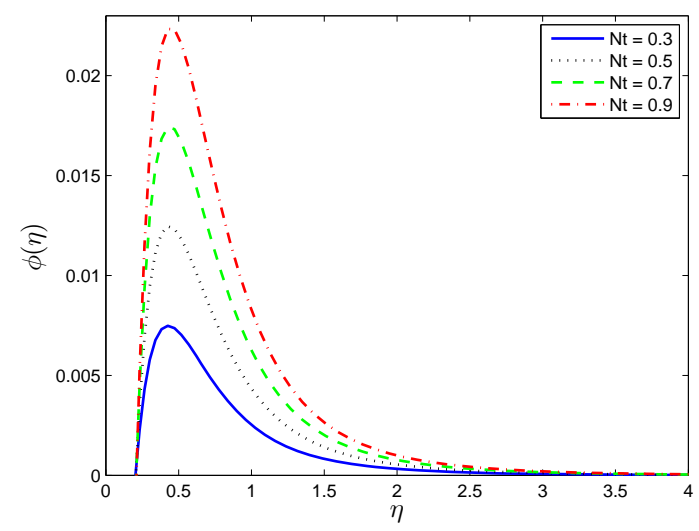

Fig. 16 Effect of the thermophoresis parameter Nt on the concentration profile $\phi(\eta)$ when $A=0.8, a=0.5, H e=0.3, E c=0.1, M=B i=$ $N_{R}=N b=\Lambda=\beta=0.5, \delta=0.1, N b=0.3, P r=10$ and $L e=1$.

is reduced due to the fact that there is an increment in the movement of cooler fluid from the surrounding fluid towards the stretching surface which leads to an increased entrainment process. The thermal boundary layer thickness is also decreased due to this effect.

The effect of the Biot number $\mathrm{Bi}$ on the temperature profile $\theta(\eta)$ is shown in Figure 13. It is noticed that increasing value of the Biot number increases the temperature profile. Increase in the Biot number causes a stronger convection and this results in higher surface temperature and increase in wall temperature values thereby leading to the temperature profile and thermal boundary layer thickness being increased. Also it was observed that increasing the Biot number indefinitely tends to reduce the convective boundary condition to the normal constant surface temperature.

The effect of the unsteadiness, Brownian motion and thermophoresis parameters on the concentration profile are displayed in Figures 14 - 16. The effect of these parameters is varied based on the incorporation of the thermophoresis and Brownian motion diffusion coefficients on the concentration boundary condition and the significance is hereby discussed below:

Figures 14 and 15 show the effect of the unsteadiness and Brownian motion parameters on the concentration profiles $\phi(\eta)$. It is noticed from these figures that increase in the values of the parameters causes a decrease in the concentration profile. Since the Brownian motion tends to intensify particle displacement away from the fluid flow regime into the surface, this effect accounts for a decrease in concentration of the nanoparticles, thus resulting in a decrease in the nanoparticle concentration boundary layer thickness.

Figure 16 depicts the effect of the thermophoresis parameter on the concentration profiles $\phi(\eta)$. Thermophoresis effect is associated with movement of nanoparticles from a hot wall to a cold wall, and since it is generated by temperature gradients, it creates a fast flow away from the moving plate, thus more fluid is heated away from the surface and this leads to an increase in the temperature within the thermal boundary layer. It is noticed that an opposite trend of the previous observations were recorded, i.e, increase in the values of the thermophoresis parameter increases the concentration profile. In addition, thermophoresis force is a phenomena which tends to create a diffusion under the effect of a temperature gradient. This increase in diffusion of the nanoparticles into the Casson nanofluid flow region leads to an increase in the concentration profile thereby increasing the nanoparticles concentration boundary layer thickness.

\section{CONCLUSION}

The effect of partial slip, convective condition and Brownian and thermophoresis diffusion coefficients, imposed on the velocity, thermal and concentration boundary conditions respectively, on the boundary layer flow of a three dimensional magnetohydrodynamic Casson nanofluid over an unsteady stretching sheet has been considered. The boundary layer equations of the fluid model are reduced to a set of nonlinear ordinary differential equations using the similarity transformation and the obtained differential equation were solved numerically for different parameters of the Casson nanofluid model. Effect of the nanofluid parameters (Lewis number, Brownian motion and thermophoresis), the Casson parameter, Biot number on the velocities, thermal and concentration boundary layers are discussed in details.

\section{ACKNOWLEDGEMENT}

The authors are thankful to the University of KwaZulu-Natal, DST-NRF Centre of Excellence in Mathematical and Statistical Sciences (CoEMaSS) and Claude Leon Foundation, South Africa for the necessary support.

\section{CONFLICT OF INTEREST}

The authors declare that there is no conflict of interest regarding the publication of this article.

\section{REFERENCES}

Bellman, R.E., Kalaba, R.E., 1965, "Quasilinearization and Nonlinear Boundary-Value Problems," Modern Analytic and Computational Methods in Science and Mathematics, Elsevier.

Bhattacharyya, K., 2013, "MHD Stagnation-Point Flow of Casson Fluid and Heat transfer over a Stretching Sheet with Thermal Radiation," Journal of Thermodynamics Article ID 169674.

https://doi.org/10.1155/2013/169674

Crane, L.J., 1970, "Flow Past a Stretching Plate," Journal of Applied Mathematical Physics(ZAMP) 21, 645-647.

https://doi.org/10.1007/BF01587695

Bhattacharyya, K., 2013, "Boundary Layer Stagnation-Point Flow of Casson Fluid and Heat Transfer Towards a Shrinking/Stretching Sheet," Frontiers in Heat and Mass Transfer 4, 2, Article ID 023003. http: //dx.doi.org/10.5098/hmt.v4.2.3003.

Kumar, B.R., Sravan Kumar, T., and Vijaya Kumar, A G., 2015, "Thermal Diffusion and Radiation Effects on Unsteady Free Convection Flow In 
the Presence of Magnetic Field Fixed Relative to the Fluid or the Plate," Frontiers in Heat and Mass Transfer 6, 12, 1-9.

http://dx.doi.org/10.5098/hmt.6.12

Ibrahim, W., Makinde, O.D., 2015, "Magnetohydrodynamic Stagnation Point Flow and Heat Transfer of Casson Nanofluid Past a Stretching Sheet with Slip and Convective Boundary Condition," Journal of Aerospace Engineering 29, 04015037.

https://doi.org/10.1061/(ASCE) AS.1943-5525. 0000529

Khan, W.A., Pop, I., 2010, "Boundary Layer Flow of a Nanofluid Past a Stretching Sheet," International Journal of Heat and Mass Transfer 53, 2477-2483.

https://doi.org/10.1016/j.ijheatmasstransfer. 2010.01 .032

Kunetsov, A.V., Nield, D.A., 2013, “The Cheng-Minkowycz Problem for Natural Convective Boundary Layer Flow in a Porous Medium Saturated by a Nanofluid: A Revised Model," International Journal of Heat and Mass Transfer 65, 682-685.

https://doi.org/10.1016/j.ijheatmasstransfer. 2013.06 .054

Kunetsov, A.V., Nield, D.A., 2014, "Natural Convective Boundary Layer Flow of a Nanofluid Past a Vertical Plate: A Revised Model," International Journal of Thermal Science 77, 126-129.

https://doi.org/10.1016/j.ijthermalsci.2013.10. 007

Makinde, O.D., Aziz A., 2011, "Boundary Layer Flow Of a Nanofluid Past a Stretching Sheet with a Convective Boundary Condition," International Journal of Thermal Sciences 50, 1326-1332.

https://doi.org/10.1016/j.ijthermalsci.2011.02. 019

Motsa, S.S., 2013, “A New Spectral Local Linearization Method for Nonlinear Boundary Layer Flow Problems," Journal of Applied mathematics Article ID 423628.

https://doi.org/10.1155/2013/423628

Motsa, S.S., Dlamini, P.G., Khumalo M., 2014, "Spectral Relaxation Method and Spectral Quasilinearization Method for Solving Unsteady Boundary Layer Flow Problems," Advances in Mathematical Physics Article ID 341964.

https://doi.org/10.1155/2014/341964

Motsa, S.S., Awad, F.G., Makukula, Z.G., Sibanda, P., 2014, “The spectral Homotopy Analysis Method Extended to Systems of Partial Differential Equations," Abstact and Applied Analysis Article ID 241594.

https://doi.org/10.1155/2014/241594

Mukhopadhyay, S., De, P.R., Bhattacharyya, K., Layek, G.C., 2013, "Casson Fluid Flow Over an Unsteady Stretching Surface," Ain Shams
Engineering Journal 4, 933-938.

https://doi.org/10.1016/j.asej.2013.04.004

Mukhopadhyay, S., 2013, "Effects of Thermal Radiation on Casson Fluid Flow and Heat Transfer Over an Unsteady Stretching Surface Subjected to Suction/Blowing," Chinese Physics B 22, 114702.

https://doi.org/10.1088/1674-1056/22/11/114702

Mustafa, M., Hayat, T., Pop, I., Hendi, A., 2011, "Stagnation-Point Flow and Heat Transfer of a Casson Fluid Towards a Stretching Sheet," Z.Naturforsch 67a, 70-76.

https://doi.org/10.5560/zna.2011-0057

Nadeem, S., Haq, R.U., Akbar, N.S., Khan, Z.H., 2013, "MHD Three-

Dimensional Casson Fluid Flow Past a Porous Linearly Stretching Sheet," Alexandria Engineering Journal 52, 577-582.

https://doi.org/10.1016/j.aej.2013.08.005

Nadeem, S., Haq, R.U., Akbar, N.S., 2014, "MHD Three-Dimensional Boundary Layer Flow of Casson Nanofluid Past a Linearly Stretching Sheet with Convective Boundary Condition," IEEE Transactions on Nanotechnology 13, 109-115.

https://doi.org/10.1109/TNANO.2013.2293735

Nandy, S.K., 2013, “Analytical Solution of MHD Stagnation-Point Flow and Heat Transfer of Casson Fluid Over a Stretching Sheet with Partial Slip," ISRN Thermodynamics Article ID 108264.

https://doi.org/10.1155/2013/108264

Noghrehabadi, A., Ghalambaz, M., Ghanbarzadeh, A., 2012, "Heat Transfer of Magnetohydrodynamic Viscous Nanofluids Over an Isothermal Stretching Sheet," Journal of Thermophysics and Heat transfer 26, 686-689.

https://doi.org/10.2514/1.T3866

Noghrehabadi, A., Pourrajab, R., Ghalambaz, M., 2012, "Effect of Partial Slip Boundary Condition on the Flow and Heat Transfer of Nanofluids Past Stretching Sheet Prescribed Constant Wall Temperature," International Journal of Thermal Sciences 54, 253-261.

https://doi.org/10.1016/j.ijthermalsci.2011.11. 017

Noghrehabadi, A., Pourrajab, R., Ghalambaz, M., 2013, "Flow and Heat Transfer of Nanofluids Over Stretching Sheet Taking into Account Partial Slip and Thermal Convective Boundary Conditions," Heat and Mass Transfer 49, 1357-1366.

https://doi.org/10.1007/s00231-013-1179-y

Sulochana, C., Ashwinkumar G.P., Sandeep, N., 2016, "Similarity solution of 3D Casson Nanofluid Flow Over a Stretching Sheet with Convective Boundary Conditions," Journal of the Nigerian Mathematical Society 35, 128-141.

https://doi.org/10.1016/j.jnnms.2016.01.001 